\title{
Induction of Hippocampal Long-Term Potentiation during Waking Leads to Increased Extrahippocampal zif-268 Expression during Ensuing Rapid-Eye-Movement Sleep
}

\author{
Sidarta Ribeiro, ${ }^{1}$ Claudio V. Mello, ${ }^{2}$ Tarciso Velho, ${ }^{2}$ Timothy J. Gardner, ${ }^{3}$ Erich D. Jarvis, ${ }^{1}$ and \\ Constantine Pavlides ${ }^{4}$ \\ ${ }^{1}$ Department of Neurobiology, Duke University Medical Center, Durham, North Carolina 27710, 2Neurological Sciences \\ Institute, Oregon Health and Science University, Beaverton, Oregon 97006, and ${ }^{3}$ Center for Physics and Biology, and \\ ${ }^{4}$ Laboratory of Neuroendocrinology, The Rockefeller University, New York, New York 10021
}

Rapid-eye-movement (REM) sleep plays a key role in the consolidation of memories acquired during waking (WK). The search for mechanisms underlying that role has revealed significant correlations in the patterns of neuronal firing, regional blood flow, and expression of the activity-dependent gene zif-268 between WK and subsequent REM sleep. Zif-268 integrates a major calcium signal transduction pathway and is implicated by several lines of evidence in activity-dependent synaptic plasticity. Here we report that the induction of hippocampal long-term potentiation (LTP) during WK in rats leads to an upregulation of zif-268 gene expression in extrahippocampal regions during subsequent REM sleep episodes. This upregulation occurs predominantly in the amygdala, entorhinal, and auditory cerebral cortices during the first REM sleep episodes after LTP induction and reaches somatosensory and motor cerebral cortices as REM sleep recurs. We also show

Mammalian sleep consists of the cyclic alternation of two behaviorally and physiologically distinct states: slow-wave sleep (SWS) and rapid-eye-movement (REM) sleep (Timo-Iaria et al., 1970; Hobson, 1995). Several lines of research indicate that sleep is important for learning (Idzikowski, 1984; Stickgold et al., 2000a, 2001; Maquet, 2001; but see Siegel, 2001). Moderate sleep deprivation impairs plasticity in the visual cortex (Frank et al., 2001) as well as the induction of hippocampal long-term potentiation (LTP) (Campbell et al., 2002), a standard model of synaptic plasticity and memory formation (Bliss and Lomo, 1973; Bliss and Collingridge, 1993). REM sleep, in particular, plays an important role in the consolidation of memories (Winson, 1985; Hennevin et al., 1995; Smith, 1996; Stickgold et al., 2000b). It has been shown that exposure to learning situations during waking

Received July 19, 2002; revised Oct. 4, 2002; accepted Oct. 4, 2002.

This work was supported by a Pew Latin American Fellowship (S.R.), a Whitehall Foundation grant (E.D.J., C.P.), a National Institute on Deafness and Other Communication Disorders grant (C.V.M.), and National Heart, Lung, and Blood Institute Grant 1 R01 HL69699-01 (C.P.). We thank D. Chialvo, C. Braham, D. Gervasoni, R. Crist, R. Costa, I. Araújo, M. Frank, S. Oster, S. Neuenschwander, and W. Singer for insightful criticism of these results and/or this manuscript; G. Oksman, J. Pantoja, M. Rivas, A. Warren-Gregory, and J. Gyrda for assistance with data collection; D. Katz for statistical advice; and M. Nicolelis, F. Nottebohm, and J. Hudspeth for their support and encouragement. This study is dedicated to Professors Gustavo de O. Castro (in memoriam) and Jonathan Winson.

Correspondence should be addressed to Sidarta Ribeiro, Department of Neurobiology, Duke University Medical Center, Box 3209, Durham, NC 27710. E-mail: ribeiro@neuro.duke.edu.

Copyright (C) 2002 Society for Neuroscience 0270-6474/02/2210914-09\$15.00/0 that hippocampal inactivation during REM sleep blocks extrahippocampal zif-268 upregulation, indicating that cortical and amygdalar zif-268 expression during REM sleep is under hippocampal control. Thus, expression of an activity-dependent gene involved in synaptic plasticity propagates gradually from the hippocampus to extrahippocampal regions as REM sleep recurs. These findings suggest that a progressive disengagement of the hippocampus and engagement of the cerebral cortex and amygdala occurs during REM sleep. They are also consistent with the view that REM sleep constitutes a privileged window for hippocampus-driven cortical activation, which may play an instructive role in the communication of memory traces from the hippocampus to the cerebral cortex.

Key words: REM sleep; zif-268; LTP; learning and memory; immediate-early gene; hippocampus; cerebral cortex; amygdala; plasticity; late sleep; early sleep

(WK) enhances subsequent REM sleep in rats (Smith and Rose, 1996). Conversely, REM sleep deprivation impairs short-term memory in rats (Pearlman and Becker, 1974; Fishbein and Gutwein, 1977; Smith and Butler, 1982; Smith and Kelly, 1988) and perceptual learning in humans (Karni et al., 1994).

Relevant WK experience results in increased hippocampal neuronal firing in rats (Pavlides and Winson, 1989; Poe et al., 2000a; Louie and Wilson, 2001) and cerebral activation in humans (Maquet et al., 2000) during subsequent REM sleep, indicating that experience-dependent brain reactivation occurs during this sleep state. In line with this notion, we have shown that brain expression of the activity-dependent gene zif-268 (same as Egr-1, NGFI-A, Krox-24, and ZENK) (Milbrandt, 1987; Sukhatme et al., 1988) is reinduced during REM sleep in rats exposed previously to an enriched environment during WK but not in unexposed controls (Ribeiro et al., 1999). Neuronal zif-268 expression is rapidly and transiently induced in response to synaptic activation via calcium signal transduction pathways (Cole et al., 1989; Wisden et al., 1990; Cullinan et al., 1995; Mello and Clayton, 1995). Therefore, the finding that $z i f-268$ is reactivated in several brain areas during REM sleep that follows a significant WK experience (Ribeiro et al., 1999) indicates that these areas are synaptically active during REM sleep.

Here we characterize the spatiotemporal dynamics of zif-268 expression during wake and sleep episodes that follow the induction of LTP in medial perforant path (mPP)-dentate gyrus (DG) synapses. We used a well established LTP-inducing, high- 
frequency stimulation (HFS) protocol (Winson and Dahl, 1986) shown previously to induce marked zif-268 upregulation in ipsilateral DG granule neurons $\sim 30$ min after stimulation (Cole et al., 1989; Wisden et al., 1990; Worley et al., 1993). Brain zif-268 expression was assessed in groups of rats representing "early" and "late" post-LTP episodes of WK, SWS, and REM sleep. We also investigated the effect of hippocampal inactivation during postLTP REM sleep on extrahippocampal expression of zif-268. The results demonstrate that zif-268 is reinduced in a hippocampusdependent manner in extrahippocampal regions during REM sleep that follows hippocampal LTP induction. Zif-268 reinduction during REM sleep may provide a window of increased extrahippocampal synaptic plasticity, possibly contributing to memory consolidation.

\section{MATERIALS AND METHODS}

Animal care. Experiments were approved by the Rockefeller University Institutional Animal Use and Care Committee, in accordance with National Institutes of Health Guidelines for the Care and Use of Laboratory Animals. Adult male Sprague Dawley rats (weight, 250-300 gm; $n=$ 4 per group; total, $n=52$ ) were housed in individual home cages, kept on a $12 \mathrm{hr}$ light/dark schedule (lights on at 7:00 A.M.), and provided with food and water ad libitum. Animals were handled daily (10-15 min) for 2 weeks before surgery, so as to effectively minimize handling and stress-related zif-268 expression (Cullinan et al., 1995; Jarvis et al., 1995; Ribeiro et al., 1999).

Electrode implant. Recording and stimulating electrodes were prepared from $125 \mu \mathrm{m}$ formvar-insulated stainless-steel wire (California Fine Wire, Grover Beach, CA) with resistances of 0.5-1.2 M $\Omega$. On the day of surgery, animals were injected with $0.1 \mathrm{mg} / \mathrm{kg}$ atropine sulfate (Baxter, Irvine, CA) to prevent excessive secretions and were deeply anesthetized $10 \mathrm{~min}$ later with $50 \mathrm{mg} / \mathrm{kg}$ sodium pentobarbital (Nembutal; Abbott Laboratories, North Chicago, IL). Animals were then placed in a rat stereotaxic device (David Kopf Instruments, Tujunga, CA). The skull was aseptically exposed, burr holes were drilled, and four stainless-steel anchor screws were inserted into the holes, two of which had plasticcoated silver wire presoldered to them (California Fine Wire), so as to provide ground and reference for stimulation and recording. Animals were implanted bilaterally with recording and stimulation electrodes in the DG $[+3.8$ anteroposterior (AP), \pm 2.1 mediolateral (ML), and -3.3 dorsoventral (DV) $\mathrm{mm}$ from bregma] and in the $\mathrm{mPP}(+7.9 \mathrm{AP}, \pm 4.1$ ML, and -3 DV from bregma), respectively (Paxinos and Watson, 1997). Electrode placement was guided by the depth profile of evoked field potentials, and the final positions of the stimulating and recording electrodes were adjusted to produce maximum responses. The electrodes and skull screw ground/reference were then connected to an 11 pin plastic plug, and the entire implant was permanently secured in place with dental acrylic cement. Animals were allowed 1 week of postsurgery recovery.

Electrophysiological recordings. Electrophysiological recordings were made using a field-effect transistor head-stage connected to the electrode plugs, and an AC/DC differential amplifier (model 3000; A-M Systems, Sequim, WA) was set at $100 \times$ gain. Evoked field potentials were bandpass filtered between $1 \mathrm{~Hz}$ and $10 \mathrm{kHz}$, digitized at $10 \mathrm{kHz}$ (MIO-16X acquisition board; National Instruments, Austin, TX), and averaged on-line using custom-built LabView software. EEG signals were bandpass filtered between 1 and $100 \mathrm{~Hz}$ and digitized at $400 \mathrm{~Hz}$ with a PowerLab recording unit (AD Instruments, Grand Junction, CO).

LTP induction. After recovery from surgery, baseline recordings of field potentials and EEG, along with handling of the animals, were performed for an additional week. Initially, an input-output function was constructed by varying the current intensity between that required to produce minimum and maximum responses (usually 20-300 $\mu \mathrm{A}$ ). A "test" intensity was then chosen that was $\sim 30 \%$ of maximum stimulation intensity. Test stimuli were delivered four times, once every $15 \mathrm{sec}$, to obtain each averaged field potential data point. Both the EPSP slope (from the initial positive rise) and the population spike amplitude of each averaged field potential were measured. Baseline recordings were taken for 1 week to ensure that the recordings were stable. On the day of the experiment, which always began within the first $2 \mathrm{hr}$ of the light cycle, animals were taken to the recording chamber, and baseline potentials were acquired for both hemispheres. After baseline field potential re- cording, one hemisphere (randomized) was subjected to HFS to produce unilateral LTP, whereas the contralateral hemisphere received no stimulation. Two HFSs per animal were applied within 5 min of each other and consisted of $1050 \mathrm{msec}$ trains of $0.25 \mathrm{msec}$ pulses at $400 \mathrm{~Hz}$, with one train every $10 \mathrm{sec}$ (Winson and Dahl, 1986). Induction of unilateral LTP was verified after the second HFS (see Fig. $1 b$ ); animals that failed to show LTP $(n=5)$ were discarded from the study and later substituted with other animals to complete all groups. An additional group of animals received HFS in one hemisphere and, as a control, lowfrequency stimulation (LFS) (10 $1 \mathrm{sec}$ trains of $0.25 \mathrm{msec}$ pulses at $10 \mathrm{~Hz}$, one train every $10 \mathrm{sec}$, same total number of pulses as in HFS) in the other hemisphere, and was assigned to the early REM sleep condition. Three control groups not subjected to HFS stimulation were also studied.

Quantitative identification of wake-sleep states. Animals were continuously observed from time of baseline recording to kill. Hippocampal EEG and evoked field potentials were also continuously recorded, and behaviors were annotated in real time on the EEG record (see Fig. 2a) according to the following categories: alert WK (active exploration of the environment with whisking and hippocampal theta rhythm), quiet WK (stillness or grooming with eyes open and non-theta low-amplitude hippocampal EEG), drowsy WK (stillness with eyes semiclosed and non-theta variable hippocampal EEG), SWS (stillness with eyes closed, plus large-amplitude slow EEG hippocampal waves), pre-REM sleep (short periods of $<10 \mathrm{sec}$ of overall stillness with brief whisking, eyes closed, and hippocampal theta rhythm), and REM sleep (overall stillness with prolonged whisking, eyes closed, and hippocampal theta rhythm). Group assignment was confirmed after an offline comparison of the behavior record and the spectrogram (see Fig. 2b), which was generated by applying a multitaper fast Fourier transform to the EEG record (Thomson, 1982). The total time spent in WK (alert plus quiet plus drowsy), SWS, and REM (pre-REM plus REM) states was calculated for each animal and averaged by group. Very mild sleep deprivation was used during selected times to standardize the kill times across groups. Specifically, all animals were kept awake in the initial $2 \mathrm{hr}$ after LTP induction, by gently tapping the recording box when animals showed drowsiness; in addition, animals in the late groups were similarly prevented from going back to sleep for $1 \mathrm{hr}$ after the first sleep cycle was completed. The criteria for assigning animals to the different wake and sleep groups were established previously (Ribeiro et al., 1999), as follows: WK animals were kept awake for $\geq 1 \mathrm{hr}$ before kill without any sleep, SWS animals had $\geq 3$ min of SWS but were woken up at the first signs of REM sleep, and REM animals were allowed to transit spontaneously from SWS into REM sleep and had $\geq 90 \mathrm{sec}$ of total REM. Figure $2 c$ depicts the mean sleep-wake structure of control and experimental groups.

Survival times and group assignment. On reaching behavioral and physiological criteria for WK, SWS, or REM sleep (see Fig. $2 a, b$ ), all animals were kept awake to prevent subsequent uncontrolled wake and/or sleep states and, thus, to normalize postcriterion experience. Zif-268 mRNA levels peak $\sim 30$ min after a given reference stimulus or behavior (Cullinan et al., 1995; Mello and Clayton, 1995; Ribeiro et al., 1999). Therefore, animals were killed by decapitation $30 \mathrm{~min}$ after reaching criteria (see Fig. 1a, asterisks), and any zif-268 expression caused by postcriteria WK was assumed to be the same across all groups. Figure $2 d$ summarizes the mean survival times relative to the time of LTP induction. Animals were assigned to one of seven groups, based on post-LTP survival times and cumulative wake-sleep states reached (see Fig. 1a). The first stimulated group (HFS/30') consisted of animals kept awake for $30 \mathrm{~min}$ after unilateral HFS and then killed; this group served as a positive control for the unilateral DG induction of zif-268 under our stimulation protocol. The six remaining stimulated groups were studied according to the cumulative wake-sleep states reached: HFS/early WK, HFS/early SWS, HFS/early REM, HFS/late WK, HFS/late SWS, and HFS/late REM. Animals in the late groups had one full sleep cycle followed by $1 \mathrm{hr}$ of WK before being randomly sorted according to wake-sleep states.

Histology and in situ hybridization. The brains were quickly removed, frozen in dry ice, and stored at $-70^{\circ} \mathrm{C}$. Serial frontal sections $(10 \mu \mathrm{m})$ were taken between approximately -1.4 and $-4.4 \mathrm{~mm}$ from bregma (Paxinos and Watson, 1997) and thaw mounted on RNase-free glass slides (Superfrost Plus; VWR Scientific, West Chester, PA) as adjacent triplicates. Thicker sections $(20 \mu \mathrm{m})$ collected every $300 \mu \mathrm{m}$ were stained with cresyl violet and used to identify the brain regions of interest. Sections were hybridized with ${ }^{35}$ S-labeled zif-268 riboprobes, as described previously (Mello et al., 1992). Absence of sense-strand hybrid- 
ization was used as a control for signal specificity. Several hybridizations were performed to optimize stringency conditions and assess consistency of results across different batches. Hybridized sets were exposed to a high-resolution ${ }^{35} \mathrm{~S}$ phosphor screen and scanned on a Storm phosphorimager system (Amersham Biosciences, Sunnyvale, CA). Selected sets were later subjected to emulsion autoradiography for cell resolution imaging of zif-268 expression. The phosphorimager autoradiograms shown in this study (see Figs. 3, 5) were obtained from an optimized hybridization batch that showed consistent expression levels across brain section triplicates. The middle section of each triplicate was chosen for blind phosphorimager densitometric quantifications with ImageQuant 5.0 software (Amersham Biosciences). Seven regions were selected for quantification based on the rat stereotaxic atlas (Paxinos and Watson, 1997): the DG, Ammon's horn (CA), entorhinal cortex (EC), dorsolateral nucleus of the amygdala ( $\mathrm{LaD}$ ), and the auditory ( $\mathrm{Au}$ ), primary somatosensory (S1), and primary motor (M1) cortices (see Fig. 3c).

Hippocampal inactivation. Hippocampal infusion of the anesthetic tetracaine was used to investigate the effects of hippocampal inactivation (Poe et al., 2000b) on extrahippocampal zif-268 expression. Different groups of animals were implanted with chronic bilateral stimulating electrodes in the mPP and recording electrodes in the DG, as described above. In addition, bilateral guide cannulas (26 gauge; Plastics One, Roanoke, VA) attached to the recording electrodes were implanted 1.5 $\mathrm{mm}$ above the DG (+3.8 AP, $\pm 2.1 \mathrm{ML}$, and $-1.8 \mathrm{DV} \mathrm{mm}$ from bregma). On the day of the experiment, tetracaine (Sigma, St. Louis, MO) was dissolved in PBS to make a $2 \%$ solution. Two microliter precision syringes (Hamilton, Reno, N V) filled with tetracaine solution and vehicle (PBS) were connected with polyethylene tubing to the infusion cannulas (33 gauge; Plastics One). Immediately before baseline recordings, the infusion cannulas were inserted into the guides with tips placed $0.5 \mathrm{~mm}$ above the recording electrodes in the DG. After baseline recordings of EEG and evoked potentials, experimental animals $(n=4)$ received bilateral HFS and were kept awake for $2 \mathrm{hr}$ before being allowed to sleep (see Fig. 5a). Ten seconds after the onset of REM sleep and without waking the animals, tetracaine $(2 \mu \mathrm{l})$ was slowly infused over 2-3 min into the left DG, while the same volume of vehicle was injected into the right DG. Tetracaine strongly reduces the EEG power recorded in the infusion site (see Fig. $5 b$ ); animals that failed to show such reduction $(n=$ 2) were discarded from the study and later substituted with other animals to complete the groups. Animals were killed 30 min after REM sleep offset, and their brains were processed for zif-268 expression. To control for nonspecific diffusion effects, animals $(n=4)$ not subjected to HFS were injected during WK with tetracaine and saline (left and right hemispheres, respectively) and killed after $30 \mathrm{~min}$ (see Fig. 5a).

\section{RESULTS}

All of the animal groups included in this study (with the exception of those subjected to LFS or hippocampus inactivation) are represented in Figure 1a, comprising three unstimulated control groups (Fig. 1a, three top bars) and seven experimental groups, the latter consisting of rats that experienced unilateral LTP induction during WK (Fig. 1a, seven bottom bars). HFS produced significant LTP that was long lasting and unilateral to the ipsilateral (stimulated) hemisphere; at $60 \mathrm{~min}$ after HFS (30 min for the HFS $/ 30^{\prime}$ group), the average EPSP slope change over baseline levels across all groups was $21.9 \pm 2.8 \%$ for ipsilateral and $-12.5 \pm 5.3 \%$ (mean \pm SEM) for contralateral (unstimulated) hemispheres. Correspondingly, the average population spike amplitude changes were $276.4 \pm 27.4 \%$ for ipsilateral and $60.1 \pm$ $15.6 \%$ for contralateral hemispheres. Post-HFS slope and spike amplitudes in ipsilateral hemispheres were significantly higher than both baseline and contralateral values (Student's $t$ test; $p<$ $0.01)$. No significant differences occurred in the amount of potentiation among groups (one-way ANOVA of slope values; $F_{(6,21)}=$ $1.17 ; p=0.36)$. Animals subjected to HFS did not display noticeable behavioral differences from unstimulated animals, having similar wake-sleep structures (Fig. 2c). Likewise, the mean survival times relative to the moment of LTP induction (Fig. $2 d$ ) did not differ across WK/SWS/REM groups (one-way ANOVA) (for

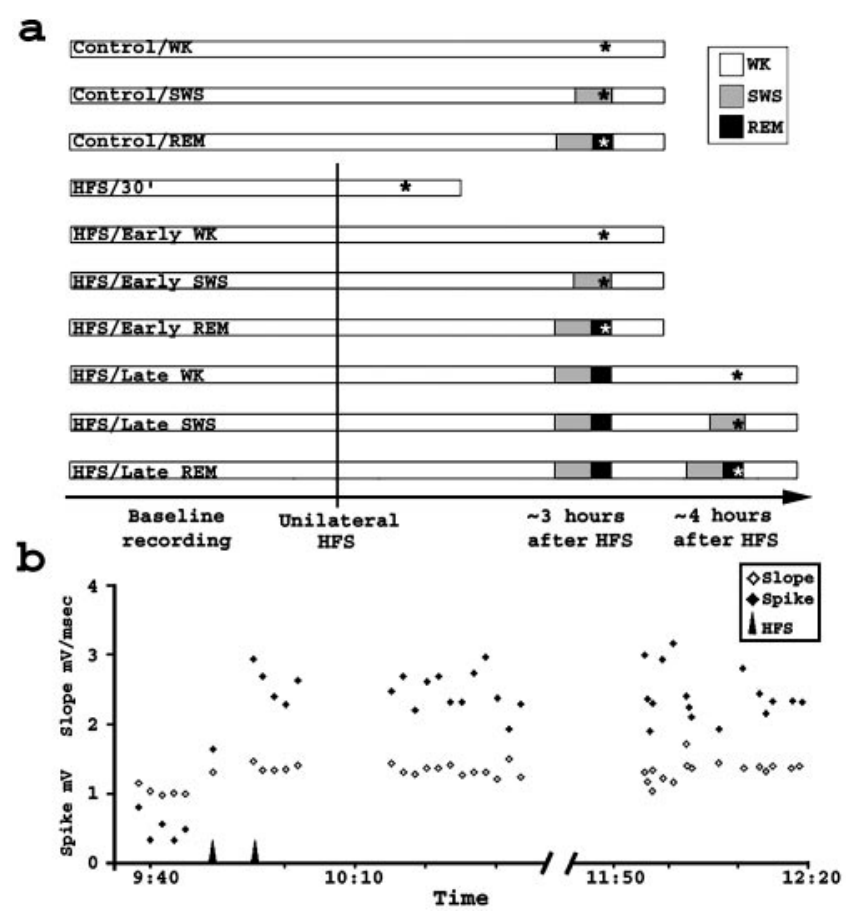

Figure 1. Experimental design and LTP induction. $a$, Seven HFS and three unstimulated control groups were studied. Acquisition of baseline field potentials was followed by HFS of one hemisphere (vertical line) to produce unilateral LTP. Group criteria are indicated by asterisks. Notice that all animals were kept awake during the last 30 min of the experiment. $b$, HFS potentiated mPP-DG synapses. A representative experiment is shown. Plotted are EPSP slope and population spike amplitudes from evoked field potentials recorded in the DG after single-pulse stimulation of the mPP. HFS (arrows) was followed by sustained potentiation of evoked responses.

early groups, $F_{(2,9)}=0.38, p=0.69$; for late groups, $F_{(2,9)}=0.89$, $p=0.44)$.

Unstimulated animals showed relatively high zif-268 expression in all brain areas studied during WK but decreased zif-268 expression when entering SWS sleep, and for some brain areas an additional decrease on entering REM sleep (see unstimulated controls in Fig. $3 b$; for quantification, see unstimulated control panels in Fig. $4 a$ ). These observations are consistent with previous findings that zif-268 expression is relatively high during WK but decreases during sleep (Pompeiano et al., 1994; Ribeiro et al., 1999). Also consistent with previous findings (Cole et al., 1989; Wisden et al., 1990; Worley et al., 1993), the induction of unilateral LTP of mPP-DG synapses led to strong zif-268 upregulation in ipsilateral DG granule neurons 30 min later (Fig. $3 a$; see Fig. $3 c$ for anatomy). Average zif-268 expression in the stimulated (ipsilateral) $\mathrm{DG}$ was $4.8 \pm 0.4$ (mean $\pm \mathrm{SEM}$ ) times higher than the average in the unstimulated (contralateral) DG.

Three hours after HFS application, zif-268 expression returned to low basal levels in the hippocampus (compare the hippocampus between Fig. $3 a$ with the HFS/early WK group in Fig. $3 b$ ) but increased bilaterally in extrahippocampal regions in all stimulated animals compared with corresponding unstimulated controls (compare cortical sites between the HFS/early WK and unstimulated controls/WK groups in Fig. 3b; for quantification, see Fig. $4 a$ ). When the animals that received HFS entered the first postLTP sleep phase, their unstimulated (contralateral) hemispheres showed a sleep-related decrease in zif-268 expression similar to the decrease found in unstimulated controls (compare unstimu- 
a

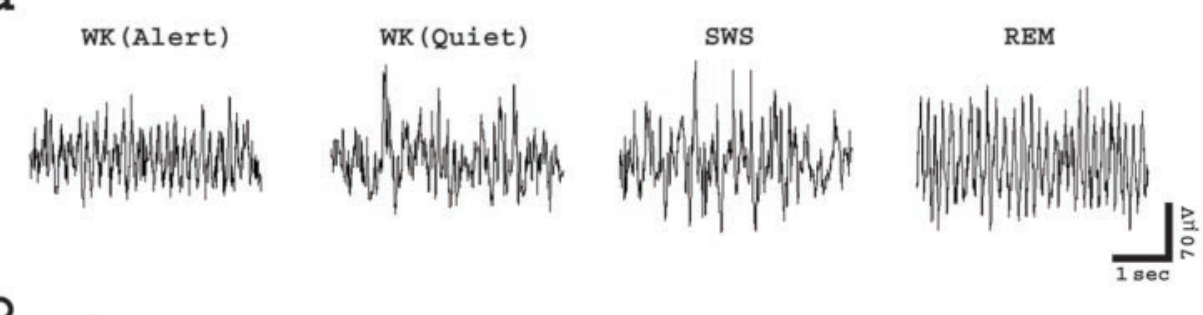

b

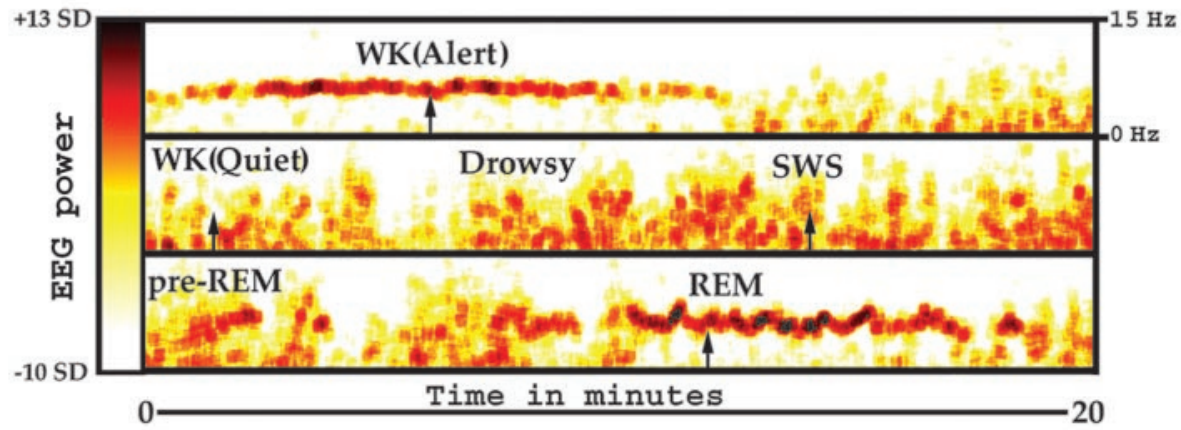

C

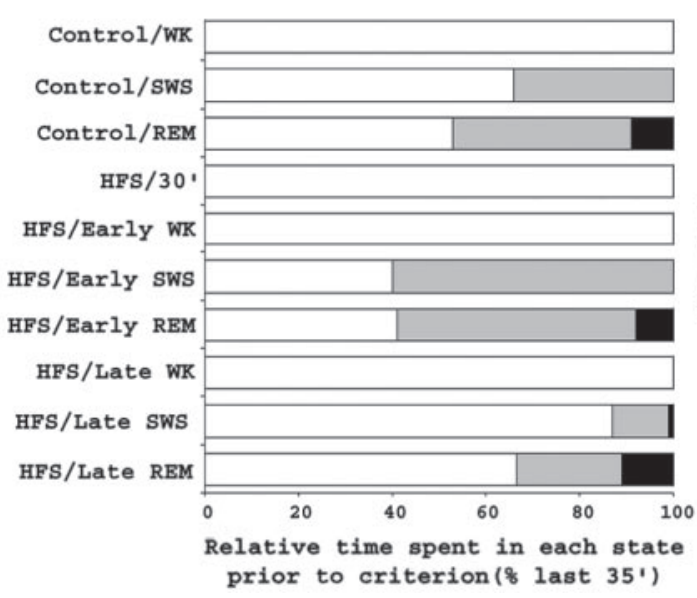

d

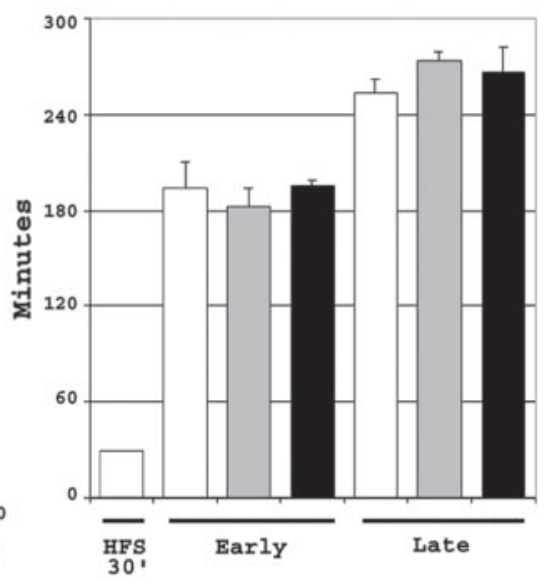

Figure 2. Assessment of wake and sleep in rats. $a$, Characteristic wake-sleep EEG traces recorded in the DG. Notice the presence of theta rhythm $(\sim 7 \mathrm{~Hz})$ during both alert WK and REM sleep states. $b$, EEG spectral analysis used for quantification of wake-sleep states. Plotted is a spectrogram (frequency and power over time) of a representative 60 min-long EEG segment. Power is coded according to the color bar to the left, which runs linearly between -10 and +13 SDs of the logarithm of the power between 6 and $9 \mathrm{~Hz}$. Frequencies are depicted in a linear scale according to the references on the top right. Arrows, Time points used to sample the EEG traces depicted in a. $c$, Wake-sleep state composition of experimental and control groups. Shown are the mean relative times spent in WK, SWS, and REM sleep, in percentage of the last $35 \mathrm{~min}$ before criterion. Histograms are colored according to the key on Figure $1 a$. $d$, Survival times after HFS. Plotted are the average elapsed times between HFS and kill (mean \pm SEM). Histograms are colored according to the key on Figure $1 a$. lated hemispheres in HFS/early groups across WK/SWS/REM sleep in Fig. $3 b$; for quantification, see the HFS/early unstimulated (contralateral) panel in Fig. $4 a$ ).

In contrast, zif-268 expression in the stimulated (ipsilateral) hemispheres of these same animals was reduced during SWS but markedly increased during REM sleep, approaching in various brain areas the same expression levels seen during WK [compare HFS hemispheres in HFS/early groups across WK/SWS/REM sleep in Fig. $3 b$; for quantification, see the HFS/early HFS (ipsilateral) panel in Fig. 4a]. Notice in Figure $4 a$ that for many brain regions, REM bars (black) have heights comparable with the WK bars (white) and higher than SWS bars (gray). Highly significant interactions were detected within left/HFS hemispheres (twoway ANOVA; $\left.F_{(48,189)}=2.48 ; p<0.000001\right)$. Although comparisons between SWS and REM groups for single brain structures did not yield significant differences when corrected for the number of comparisons, grouping the structures in three putative anatomical divisions (hippocampus, DG plus CA; proximal extrahippocampal, EC plus Au plus LaD; distal extrahippocampal, S1 plus M1) revealed significantly higher zif-268 levels in REM sleep than in SWS (Bonferroni post hoc tests, $* p<0.05$ ) in proximal extrahippocampal regions (Fig. $4 a$ ). This unilateral in- crease in zif-268 expression during REM sleep in HFS animals can be clearly seen when expressed as an interhemispheric (HFS/ unstimulated) ratio, as in Figure $4 b$. Although no appreciable interhemispheric differences in zif-268 expression were seen in unstimulated controls (left/right ratios of $\sim 1$ ), HFS/early animals showed significantly higher interhemispheric zif-268 expression ratios (HFS/unstimulated ratios significantly $>1$ ) during REM sleep in the EC and Au. This extrahippocampal upregulation of zif-268 during REM sleep was restricted to a coronal band of $\sim 500 \mu \mathrm{m}$ around the hippocampal site of LTP induction (DG) and was robust in all layers of the cortex, except for layer IV, which showed low levels of gene expression (Fig. $3 d$ ).

These data indicate that zif-268 expression is reinduced in the brain during the first REM sleep episode that follows HFS of the hippocampus during WK. Because the interval between the application of HFS and kill did not differ across WK/SWS/REM sleep groups (Fig. 2d), the observed effect cannot be explained by differences in the survival intervals after stimulation. Additional controls ( $n=4$; data not shown) that received HFS in one hemisphere and LFS in the other hemisphere showed no significant difference in gene expression profile from that of HFS/ unstimulated animals (two-way ANOVA; $F_{(6,42)}=1.8 ; p=0.12$ ), 


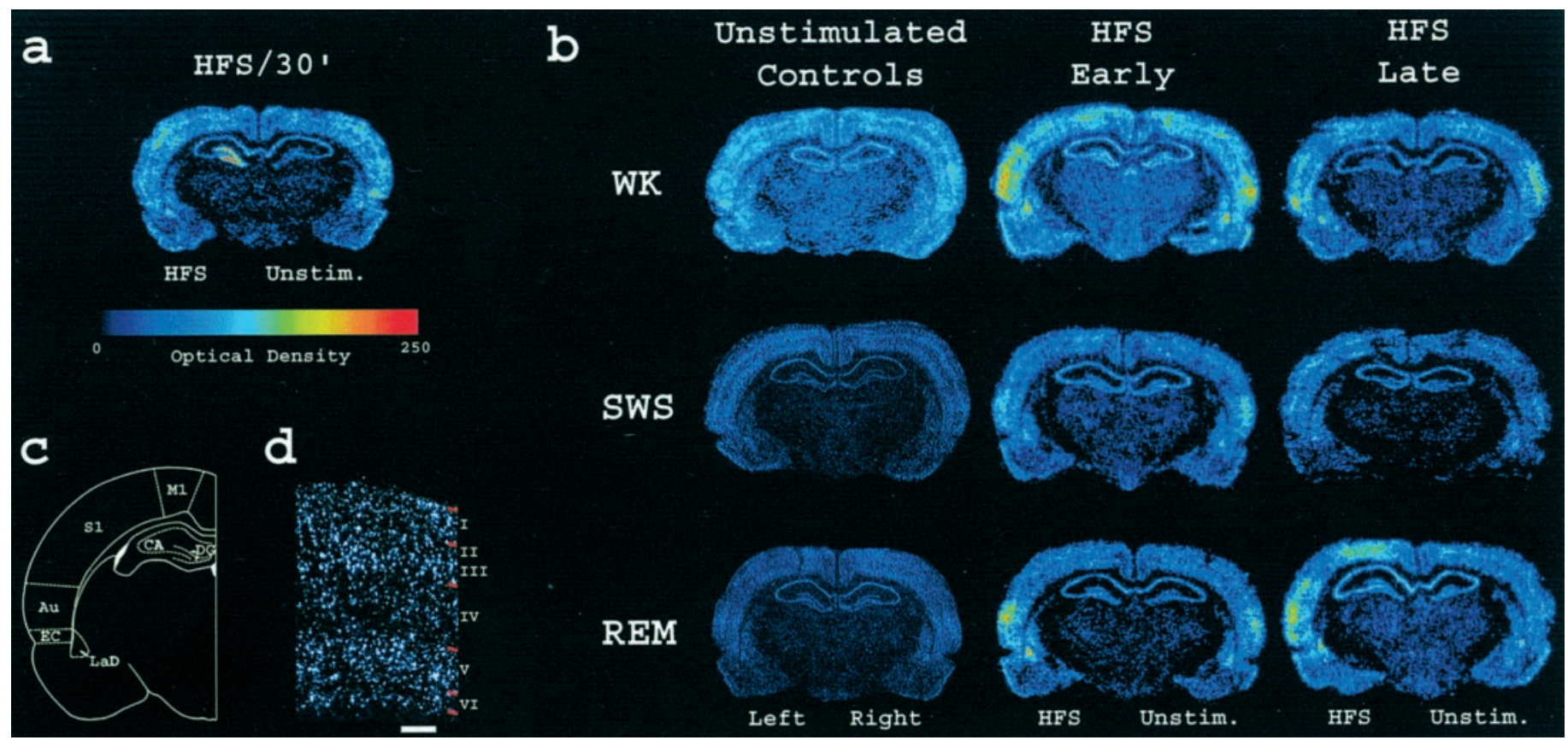

Figure 3. Post-LTP zif-268 expression profile across wake-sleep cycles. a, Phosphorimager autoradiogram of a representative zif-268-hybridized section. The ipsilateral (but not contralateral) DG showed marked zif-268 upregulation 30 min after unilateral HFS. $b$, Zif-268 expression levels in the brain during early and late WK, SWS, and REM sleep depend on previous WK stimulation. Both HFS-stimulated and unstimulated controls showed a generalized decrease in brain zif-268 expression in association with SWS, whereas HFS-stimulated hemispheres in both HFS early and late groups showed a selective increase in zif-268 expression in the cortex and amygdala in association with REM sleep. $c$, Brain regions in which zif-268 expression was quantified. $d$, Zif-268 cortical reinduction during REM sleep occurred predominantly in layers II, III, and V. Shown is a dark-field view of the auditory cortex in a representative section from the HFS/early REM group, hybridized to zif-268 and exposed to autoradiographic emulsion. White-silver grains denote gene expression; red marks on the right indicate cell layer boundaries, as determined by cresyl violet counterstaining. Scale bar, $250 \mu \mathrm{m}$.

Figure 4. Quantification of zif-268 expression across the wake-sleep cycle. $a$, Absolute levels of zif-268 expression. Both hemispheres of unstimulated controls showed decreased zif-268 expression levels during SWS and REM sleep compared with WK. The same general pattern was observed in the unstimulated (contralateral) hemispheres of HFS animals. In contrast, several brain structures in the HFS (ipsilateral) hemispheres of both HFS/early and HFS/late groups showed high zif-268 expression during WK and REM sleep and low zif-268 expression during SWS. Zif-268 reinduction occurred in proximal extrahippocampal regions (EC, $\mathrm{Au}$, and $\mathrm{LaD}$ ) during Early REM sleep and reached distal extrahippocampal areas (S1 and M1) during Late REM sleep (Bonferroni post hoc tests; $\left.{ }^{*} p<0.05\right)$. OD, Optical density. $b$, Interhemispheric zif-268 expression ratios. Histograms are according to the key in $a$. Whereas unstimulated controls did not show differences between left and right

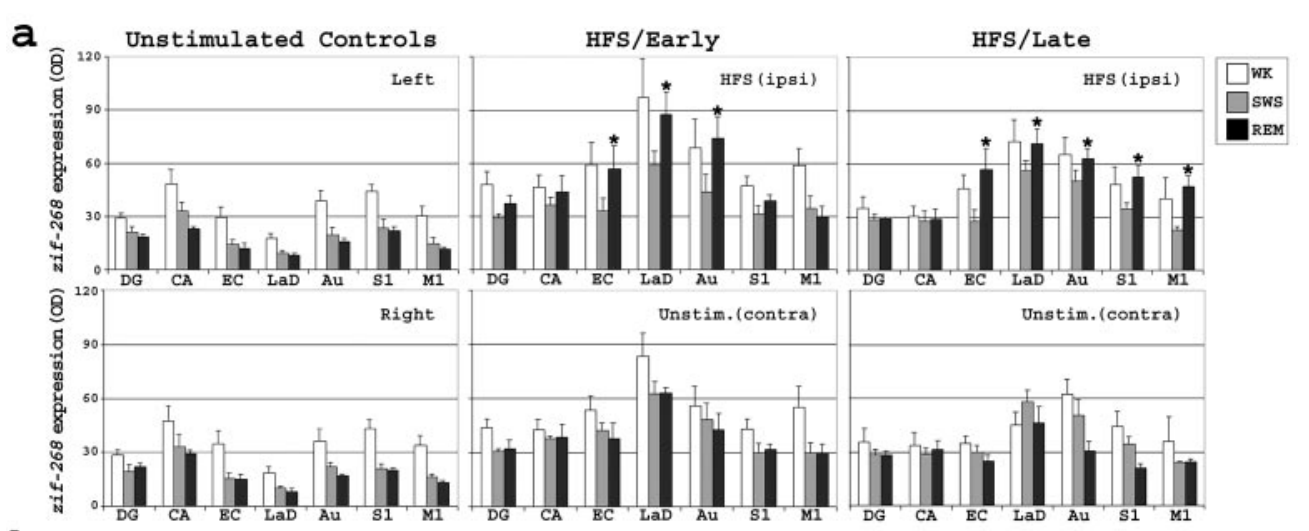

b

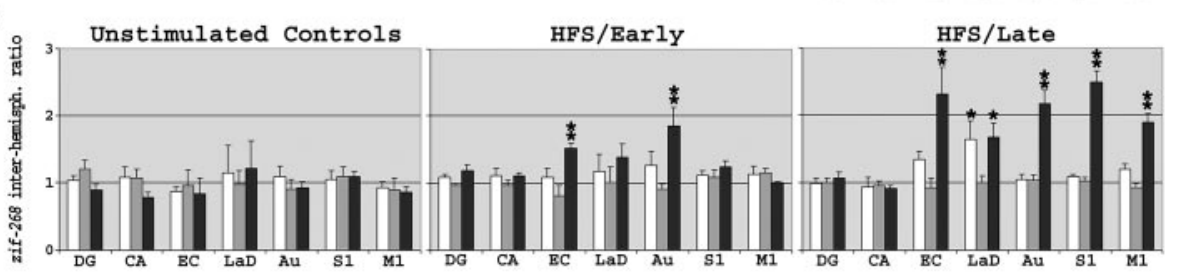

hemispheres (two-way ANOVA; $F_{(12,63)}=0.33 ; p=0.98$ ), significant interactions were detected in HFS groups (two-way ANOVA; $F_{(30,126)}=3.72 ; p<$ 0.0001), because of higher zif-268 interhemispheric ratios (HFS/unstimulated) in the EC, LaD, Au, S1, and M1 during REM sleep than during preceding SWS (Bonferroni post hoc tests; ${ }^{*} p<0.05 ;{ }^{*} p<0.01$ ). Notice that the interhemispheric zif-268 expression ratio was also significantly higher for LaD in the HFS/late WK group than in the HFS/late SWS group.

indicating that specific patterns of WK electrical stimulation, such as HFS, are required for the reinduction of zif-268 during ensuing REM sleep. Notably, HFS of the mPP induces both zif-268 primary induction and LTP in the DG, whereas LFS fails to produce either effect (Cole et al., 1989).
Essentially the same effects as described above for early groups were observed in the late groups: animals showed a generalized sleep-related decrease in brain zif-268 expression in unstimulated (contralateral) hemispheres but a selective second upregulation of zif-268 expression in extrahippocampal sites of stimulated 
a
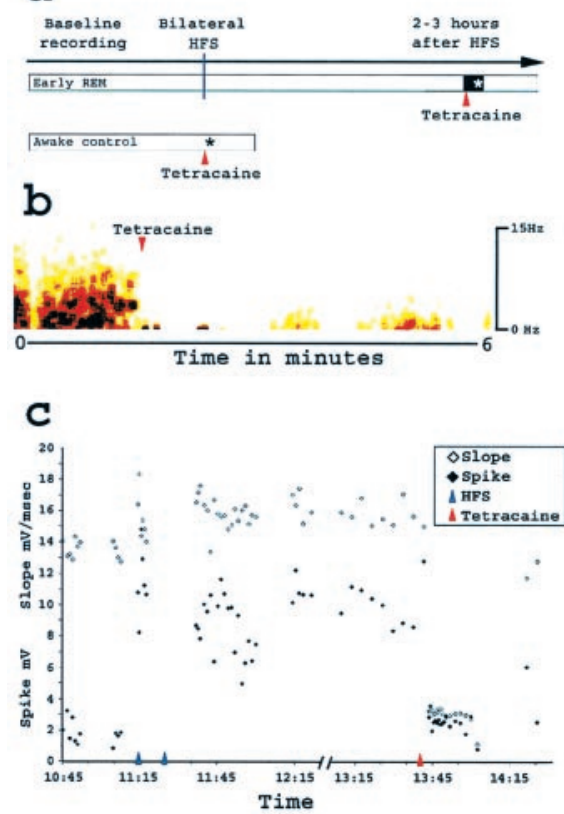
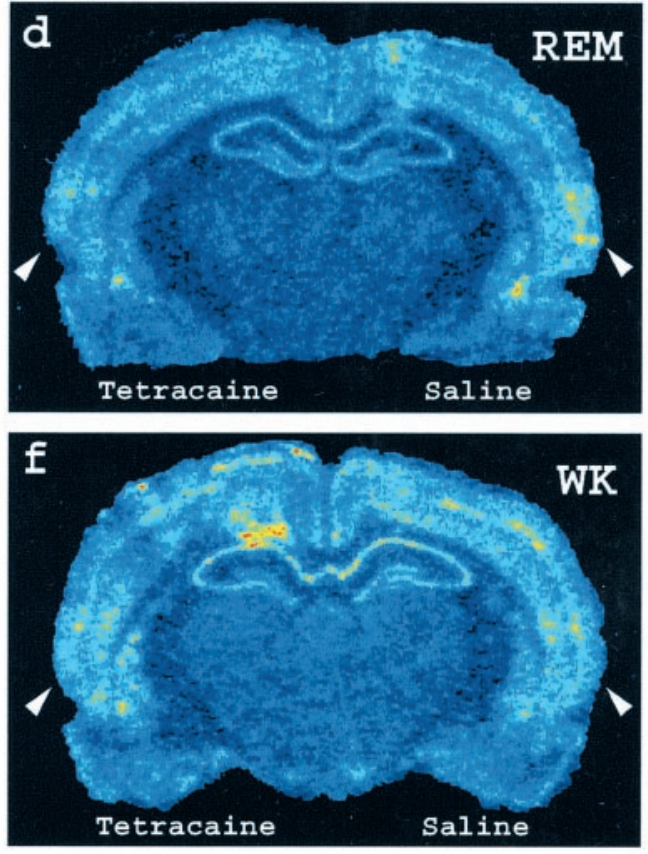
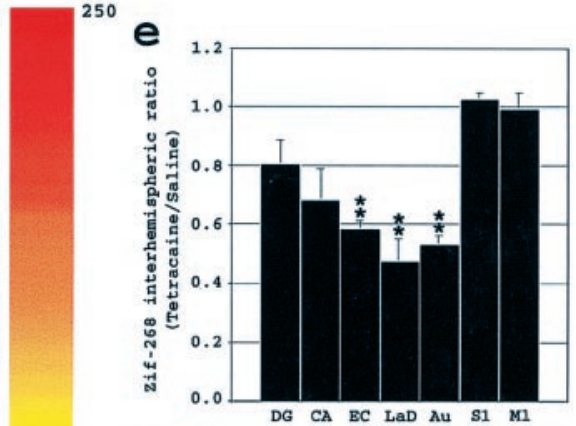

g

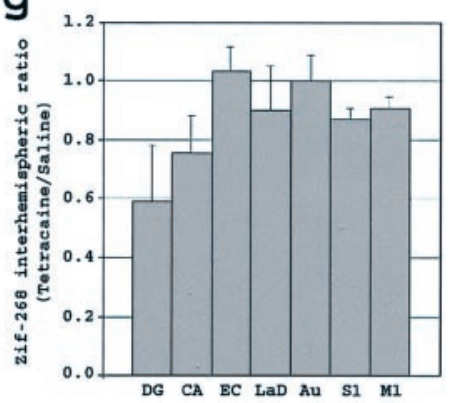

Figure 5. Effect on extrahippocampal zif-268 expression of hippocampal inactivation during REM sleep and WK. $a$, Experimental design of the hippocampal inactivation experiment (see Materials and Methods). $b$, Tetracaine infusion strongly reduced EEG power in the DG immediately after the beginning of injection. Amplitude is color coded as in Figure 2b. $c$, Tetracaine effects on evoked potentials. A representative experiment is shown; notice that potentials almost recovered to previous potentiated levels right before kill, at 14:15. $d$, Zif-268 brain expression levels during early REM sleep, after bilateral HFS and unilateral tetracaine inf usion. Shown is a phosphorimager autoradiogram of a representative brain section hybridized for zif-268. There was a marked reduction of zif-268 expression in the left EC, Au, and $\mathrm{LaD}$ (tetracaine side) compared with the right hemisphere (saline side). Arrowheads, Auditory cortex (see Fig. $3 c$ for anatomical localization). e, Zif-268 expression interhemispheric ratios (tetracaine/saline) in bilateral HFS and early REM animals $\left({ }^{*} p<0.05\right.$ and $\left.{ }^{* *} p<0.01\right)$. $f$, Zif- 268 brain expression levels during WK, after unilateral tetracaine inf usion. Zif-268 expression levels were comparable between hemispheres. Arrowheads, Auditory cortex. g, Zif-268 expression interhemispheric ratios (tetracaine/saline) in WK control animals.

hemispheres during a second post-LTP REM sleep episode (see HFS/late groups in Figs. $3 b, 4 a$ ). Whereas reactivation of zif-268 expression during early REM sleep was most robust in proximal extrahippocampal regions (EC, $\mathrm{LaD}$, and $\mathrm{Au}$ ), during late REM sleep the effect also included distal extrahippocampal areas (S1 and M1) (Fig. 4a). This is particularly clear when interhemispheric zif-268 expression ratios are calculated (Fig. 4b, HFS/late panel).

The fact that most of the REM-associated reinduction of zif268 expression occurs in extrahippocampal sites raised the question of whether extrahippocampal zif-268 reinduction during REM sleep is dependent on concurrent hippocampal activity. To investigate this issue, we assessed whether unilateral inactivation of the hippocampus during post-LTP early REM sleep disrupts zif-268 reinduction in proximal extrahippocampal regions. Rats $(n=4)$ were subjected to bilateral HFS to induce comparable LTP in both hemispheres (average population spike change of $582.3 \pm 70.9 \%$ and $422.2 \pm 167.1 \%$ for left and right hemispheres, respectively; mean \pm SEM). The animals were kept awake for a minimum of $2 \mathrm{hr}$ and then allowed to sleep (Fig. $5 a$ ). Shortly after the onset of REM sleep (duration of $2.4 \pm 0.7 \mathrm{~min}$; mean \pm SEM), left hippocampi were inactivated with tetracaine, whereas right hippocampi received saline. Animals were killed 30 min after REM sleep offset, and their brains were processed for zif-268 expression. Unstimulated controls $(n=4)$ similarly injected during WK were also analyzed (Fig. $5 a$ ).

Tetracaine administration strongly reduced the EEG power in hemispheres ipsilateral to the anesthetic injection (Fig. 5b). An immediate and robust reduction of evoked potentials was also observed in the tetracaine-infused (left) DG (Fig. 5c) (population spike amplitude decreased by $73.1 \pm 11.6 \%$; mean \pm SEM), whereas minor effects were observed in the vehicle-infused (right) DG (population spike amplitude decreased by $3.6 \pm 2.6 \%$; mean \pm SEM). Tetracaine effects lasted for $\sim 30$ min, keeping the hippocampus silenced during the entire REM sleep episode and most of the ensuing WK. These results confirm the effectiveness of the tetracaine treatment to locally inactivate the hippocampus during a restricted time window (Poe et al., 2000b), with very interesting effects on zif-268 expression. Although the salineinjected (right) hemispheres showed the expected REM-related high expression of zif-268 in the cerebral cortex (EC and $\mathrm{Au}$ ) and amygdala (LaD) (Fig. 5d, compare saline-injected hemisphere with HFS hemisphere in REM/early group, Fig. $3 b$ ), the increase in zif-268 expression was blocked in those same regions in the tetracaine-injected (left) hemispheres (Fig. $5 d$ ). This effect becomes very clear when expressed as the interhemispheric zif-268 expression ratio, indicating significantly lower zif-268 expression in tetracaine-injected than in saline-injected hemispheres (ratio values are significantly $<1$ for the $\mathrm{EC}, \mathrm{Au}$, and $\mathrm{LaD}$ in Fig. 5e) (one-way ANOVA; $F_{(6,21)}=13.72 ; p<0.0001$; followed by Bonferroni post hoc tests). No effect on zif-268 expression in these same areas was seen when comparable hippocampal tetracaine injections were applied during WK (Fig. 5f,g) (one-way ANOVA; $\left.F_{(6,21)}=1.78 ; p=0.15\right)$. Thus, extrahippocampal zif-268 expression during WK is primarily independent of hippocampal activity, presumably because of the intense thalamocortical processing 

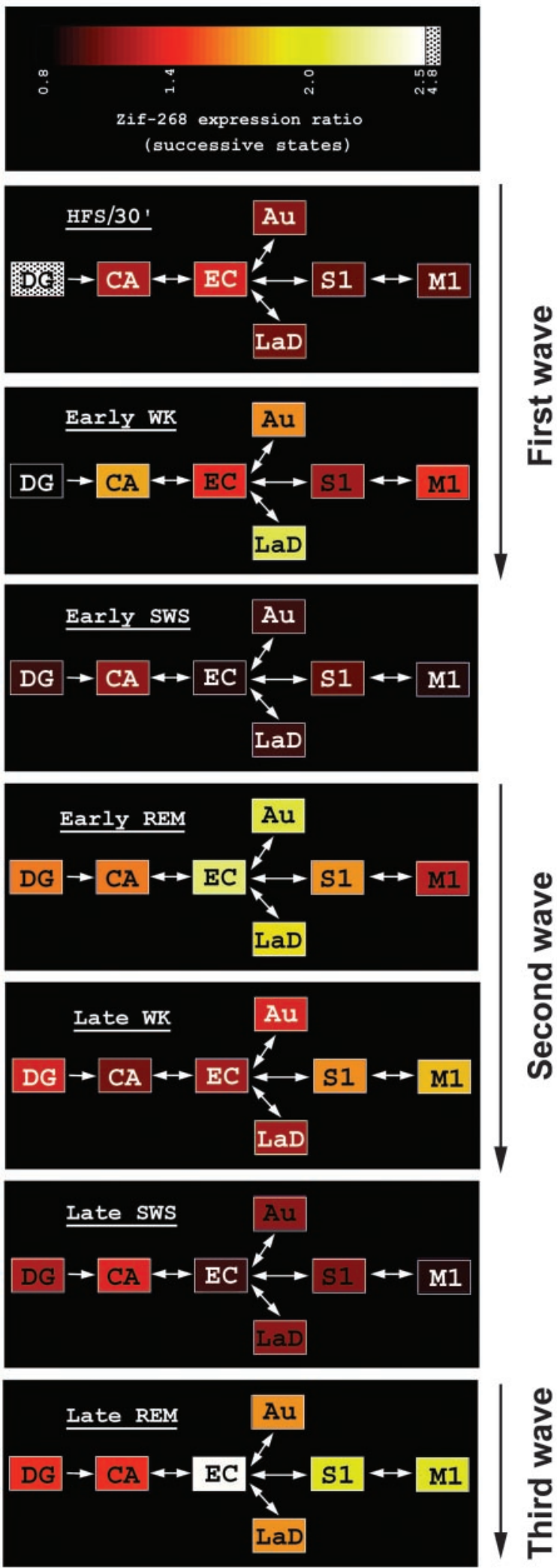

Figure 6. Hippocampal LTP is followed by a series of zif-268 induction waves that progressively disengage the hippocampus and engage the cerebral cortex and the amygdala as the wake-sleep cycle recurs. Shown is a diagram of zif-268 expression across states, organized according to the intrinsic and extrinsic connectivity of the hippocampus with brain areas showing zif-268 reinduction. The mean ratios of zif-268 expression across successive states (HFS/30' over control/WK; HFS/early WK over HFS/ that characterizes WK. This also indicates that the ipsilateral blockade of zif-268 reinduction during REM sleep in the cortex and amygdala of tetracaine-injected animals cannot be explained by diffusion of tetracaine from the injection site. Therefore, extrahippocampal zif-268 reinduction during early REM sleep requires an active hippocampus.

\section{DISCUSSION}

\section{Experience-dependent zif-268 reinduction during REM sleep}

We have shown for the first time that: (1) zif-268 is reinduced during REM sleep in extrahippocampal regions after the induction of hippocampal LTP during WK; (2) zif-268 reinduction occurs predominantly in the amygdala, entorhinal, and auditory cortices during the first REM sleep episodes after LTP induction and reaches somatosensory and motor cortices as REM sleep recurs; and (3) extrahippocampal zif-268 reinduction during REM sleep requires concurrent hippocampal activity. These results extend our previous report that zif-268 is upregulated during REM sleep in the brains of animals exposed previously to a novel enriched environment but not in unstimulated controls (Ribeiro et al., 1999). The LTP-inducing HFS protocol provided a spatially more discrete and standardized stimulation paradigm, inducing gene expression in a more specific anatomical pathway downstream of the stimulation site, compared with the widespread brain activity associated with the enriched environment paradigm. The stimulation was also more discrete temporally, allowing a better dissection of the kinetics of gene regulation.

Our results are seemingly at odds with several studies showing that sleep downregulates the expression of immediate-early genes (IEG) (Pompeiano et al., 1992, 1994; O'Hara et al., 1993; Basheer et al., 1997; Cirelli and Tononi, 1998). Two factors might explain this discrepancy. First, these studies did not attempt to separate the specific contributions of SWS and REM sleep to IEG expression. We have found that indeed a marked decrease in zif-268 expression occurs during SWS, regardless of the preceding WK experience. Therefore, the postsleep zif-268 downregulation reported previously (O'Hara et al., 1993; Pompeiano et al., 1994; Cirelli and Tononi, 1998) likely reflects the natural predominance of SWS over REM sleep in spontaneous sleep episodes (TimoIaria et al., 1970; Hobson, 1995). Second and perhaps more importantly, those studies did not examine the effect of exposing animals to relevant WK experience a few hours before the sleep episodes investigated. Thus, their results likely correspond to the zif-268 downregulation that we find in association with SWS and REM sleep in unstimulated control groups (Figs. 3b, 4a).

Another important point regards the mechanisms underlying experience-dependent zif-268 upregulation during REM sleep. Cirelli et al. (1996) have shown that IEG expression in the brain is dependent on the integrity of the noradrenergic nucleus locus ceruleus, leading the authors to propose that a general decrease in neuronal excitability attributable to the silencing of the locus

30 '; HFS/early SWS over HFS/early WK, etc.) are plotted in color according to the key at the top. Panels are labeled with the name of the later state. Boxes, Brain regions; arrows, connections between them. Three distinct waves of experience-dependent zif-268 upregulation generated during REM sleep or WK and separated by periods of SWS-associated gene downregulation were observed. 
ceruleus during sleep (Aston-Jones and Bloom, 1981) causes a sleep-related decrease in IEG expression. This rationale accounts well for the SWS/REM sleep downregulation of zif-268 observed in unstimulated animals but not for the gene upregulation seen during REM sleep in exposed animals. At least three hypotheses can be formulated to reconcile the lack of locus ceruleus activity during REM sleep with concurrent zif-268 upregulation. The most parsimonious regards cholinergic transmission, which is very robust during REM sleep (Hobson, 1992; Williams et al., 1994) and could in principle compensate for the lack of noradrenaline, setting in motion molecular cascades that would result in the upregulation of zif-268 (Greenberg et al., 1986; Shiromani et al., 1992). Another possibility is that the locus ceruleus actually releases noradrenaline during REM sleep that follows relevant WK experience, either via subthreshold activity or through experience-dependent neuronal firing. Although less likely than the previous one, this specific hypothesis has yet to be tested. Finally, it is possible that zif-268 reinduction during REM sleep is triggered in an experience-dependent manner during the transition between REM sleep and subsequent WK, a brief moment in which REM-associated neuronal activity and noradrenaline release might coexist in space and time.

\section{Hippocampal interactions with the cerebral cortex and amygdala during REM sleep}

Considering the known neuroanatomical circuitry downstream of the DG (Lopes da Silva et al., 1990; Paxinos, 1995), our results reveal a sequence of three spatiotemporally distinct waves of zif-268 upregulation after the induction of LTP (Fig. 6). The first begins locally at the stimulation site (DG) $\sim 30$ min after the application of HFS, reaches proximal brain areas relative to the stimulation site (CA, $\mathrm{Au}$, and $\mathrm{LaD}$ ) after $3 \mathrm{hr}$ of sustained wakefulness (early WK), and is terminated during early SWS. A second wave begins during early REM sleep predominantly in brain regions proximal to the stimulus site (EC, $\mathrm{Au}$, and $\mathrm{LaD})$, propagates to distal brain regions (S1 and M1) during late WK, and is terminated during late SWS. Finally, a third wave of zif-268 upregulation begins during late REM sleep in all proximal (EC, $\mathrm{Au}$, and $\mathrm{LaD}$ ) and distal (S1 and M1) extrahippocampal regions studied. Although later time points were not investigated, our data suggest that this third wave extends through WK that follows late REM sleep, being terminated during ensuing SWS. Notice that hippocampal regions (DG and CA) show a gradual decrease in zif-268 expression from the first to the second and third waves. Conversely, the most distal extrahippocampal region studied (M1), several synapses away from the site of HFS application, shows an opposite gene expression profile: a gradual increase in zif-268 expression from the first to the second and third waves. These results indicate that hippocampal LTP induction during WK leads to a series of zif-268 upregulation waves that propagate from the hippocampus to cortical areas during the course of WK and REM sleep but that are terminated during SWS.

To the extent that zif-268 expression reflects synaptic activation occurring during a restricted time window before kill, we provide evidence that a significant increase in synaptic activity occurs in several extrahippocampal brain areas during REM sleep that follows induction of hippocampal LTP. Furthermore, extrahippocampal activation during REM sleep is dependent on concurrent hippocampal activity, suggesting the existence of a hippocampofugal process of cortical and amygdalar activation during REM sleep. Although additional experimentation is required to determine whether distal zif-268 upregulation during late REM sleep depends on proximal gene upregulation during early REM sleep, the spatiotemporal pattern of zif-268 expression and the effects of hippocampal inactivation on proximal extrahippocampal zif-268 expression suggest that brain reactivation during REM sleep occurs sequentially, initially in brain regions proximal to the hippocampus and later reaching more distal sites.

Independent lines of evidence show that although several kinds of memory initially depend on the hippocampus, such memories gradually become hippocampus-independent and correspondingly more reliant on the cerebral cortex for long-term storage (Scoville and Milner, 1957; Mishkin, 1978; Kesner and Novak, 1982; Squire, 1992; Izquierdo and Medina, 1997; Bontempi et al., 1999). Based on our findings, we suggest that REM sleep may constitute a privileged window for hippocampus-driven cortical activation, free from waking interference, and, in principle, capable of playing an instructive role in the communication of memory traces from the hippocampus to the cortex. The fact that zif-268 reinduction is anatomically more extensive in late than in early REM sleep is especially interesting considering that late but not early post-training REM episodes are crucial for learning (Smith and Rose, 1996; Stickgold et al., 2000b). It is also noteworthy that the LaD shows significantly high levels of zif-268 expression during late WK and REM. This observation adds to the evidence that the amygdala plays a sustained role in information processing after the initial phase of LTP or behavioral learning (Ben-Ari and Le Gal la Salle, 1972; Izquierdo and Medina, 1997; Rogan et al., 1997; McGaugh, 2000).

Although the exact relationship between brain activation and ensuing activity-dependent gene expression is still a matter of some debate, it is important to note that zif-268 encodes a transcriptional regulator (Milbrandt, 1987; Sukhatme et al., 1988) that controls the expression of several target genes, some of which are involved in various aspects of neuronal function and plasticity. For example, zif-268 regulates the expression of synapsins (Thiel et al., 1994; Petersohn et al., 1995), the most abundant proteins of synapses, with a key function in neurotransmission (De Camilli, 1995). By coupling neuronal activation to the upregulation of cell components such as synapsins, zif-268 may play an important role in the regulation of activity-dependent synaptic plasticity. This notion is supported by the fact that zif-268 expression is increased in brain regions that undergo dendritic changes after exposure to an enriched environment (Wallace et al., 1995) and in association with novelty/learning behavioral paradigms (Mello et al., 1992; Nikolaev et al., 1992; Jarvis et al., 1995) and hippocampal LTP induction (Cole et al., 1989; Wisden et al., 1990). Most compellingly, zif-268 expression has been shown to be necessary for the long-term maintenance of hippocampal LTP and different types of spatial and nonspatial memories (Jones et al., 2001). Because the consolidation of memories most likely requires modification, addition, and/or extinction of synapses (Bliss and Collingridge, 1993), it is plausible that experience-dependent brain reactivation during REM sleep (Pavlides and Winson, 1989; Maquet et al., 2000; Poe et al., 2000a; Louie and Wilson, 2001) contributes to the formation of long-lasting memories through the upregulation of activity-dependent genes associated with synaptic plasticity. More specifically, hippocampofugal zif-268 upregulation during REM sleep may reflect the propagation of calcium-dependent postsynaptic changes from the hippocampus to cortical and amygdalar networks, seemingly a crucial step for the consolidation of long-term memories (Frankland et al., 2001). 


\section{REFERENCES}

Aston-Jones G, Bloom FE (1981) Activity of norepinephrine-containing locus ceruleus neurons in behaving rats anticipates fluctuations in the sleep-waking cycle. J Neurosci 1:876-886.

Basheer R, Sherin JE, Saper CB, Morgan JI, McCarley RW, Shiromani PJ (1997) Effects of sleep on wake-induced c-fos expression. J Neurosci 17:9746-9750.

Ben-Ari Y, Le Gal la Salle G (1972) Plasticity at unitary level. II. Modifications during sensory-sensory association procedures. Electroencephalogr Clin Neurophysiol 32:667-679.

Bliss TV, Collingridge GL (1993) A synaptic model of memory: longterm potentiation in the hippocampus. Nature 361:31-39.

Bliss TV, Lomo T (1973) Long-lasting potentiation of synaptic transmission in the dentate area of the anaesthetized rabbit following stimulation of the perforant path. J Physiol (Lond) 232:331-356.

Bontempi B, Laurent-Demir C, Destrade C, Jaffard R (1999) Timedependent reorganization of brain circuitry underlying long-term memory storage. Nature 400:671-675.

Campbell IG, Guinan MJ, Horowitz JM (2002) Sleep deprivation impairs long-term potentiation in rat hippocampal slices. J Neurophysiol 88:1073-1076.

Cirelli C, Tononi G (1998) Differences in gene expression between sleep and waking as revealed by mRNA differential display. Brain Res Mol Brain Res 56:293-305.

Cirelli C, Pompeiano M, Tononi G (1996) Neuronal gene expression in the waking state: a role for the locus coeruleus. Science 274:1211-1215.

Cole AJ, Saffen DW, Baraban JM, Worley PF (1989) Rapid increase of an immediate early gene messenger RNA in hippocampal neurons by synaptic NMDA receptor activation. Nature 340:474-476.

Cullinan WE, Herman JP, Battaglia DF, Akil H, Watson SJ (1995) Pattern and time course of immediate early gene expression in rat brain following acute stress. Neuroscience 64:477-505.

De Camilli P (1995) Neurotransmission. Keeping synapses up to speed. Nature 375:450-451.

Fishbein W, Gutwein BM (1977) Paradoxical sleep and memory storage processes. Behav Biol 19:425-464.

Frank MG, Issa NP, Stryker MP (2001) Sleep enhances plasticity in the developing visual cortex. Neuron 30:275-287.

Frankland PW, O'Brien C, Ohno M, Kirkwood A, Silva AJ (2001) $\alpha$-CaMKII-dependent plasticity in the cortex is required for permanent memory. Nature 411:309-313.

Greenberg ME, Ziff EB, Greene LA (1986) Stimulation of neuronal acetylcholine receptors induces rapid gene transcription. Science 234:80-83.

Hennevin E, Hars B, Maho C, Bloch V (1995) Processing of learned information in paradoxical sleep: relevance for memory. Behav Brain Res 69:125-135.

Hobson JA (1992) Sleep and dreaming: induction and mediation of REM sleep by cholinergic mechanisms. Curr Opin Neurobiol 2:759-763

Hobson JA (1995) Sleep. Scientific American Library. New York: Freeman.

Idzikowski C (1984) Sleep and memory. Br J Psychol 75:439-449.

Izquierdo I, Medina JH (1997) Memory formation: the sequence of biochemical events in the hippocampus and its connection to activity in other brain structures. Neurobiol Learn Mem 68:285-316.

Jarvis ED, Mello CV, Nottebohm F (1995) Associative learning and stimulus novelty influence the song-induced expression of an immediate early gene in the canary forebrain. Learn Mem 2:62-80.

Jones MW, Errington ML, French PJ, Fine A, Bliss TV, Garel S, Charnay P, Bozon B, Laroche S, Davis S (2001) A requirement for the immediate early gene Zif268 in the expression of late LTP and long-term memories. Nat Neurosci 4:289-296.

Karni A, Tanne D, Rubenstein BS, Askenasy JJ, Sagi D (1994) Dependence on REM sleep of overnight improvement of a perceptual skill. Science 265:679-682.

Kesner RP, Novak JM (1982) Serial position curve in rats: role of the dorsal hippocampus. Science 218:173-175.

Lopes da Silva FH, Witter MP, Boeijinga PH, Lohman AH (1990) Anatomic organization and physiology of the limbic cortex. Physiol Rev 70:453-511.

Louie K, Wilson MA (2001) Temporally structured replay of awake hippocampal ensemble activity during rapid eye movement sleep. Neuron 29:145-156.

Maquet P (2001) The role of sleep in learning and memory. Science 294:1048-1052.

Maquet P, Laureys S, Peigneux P, Fuchs S, Petiau C, Phillips C, Aerts J, Del Fiore G, Degueldre C, Meulemans T, Luxen A, Franck G, Van Der Linden M, Smith C, Cleeremans A (2000) Experience-dependent changes in cerebral activation during human REM sleep. Nat Neurosci 3:831-836.
McGaugh JL (2000) Memory-a century of consolidation. Science 287:248-251.

Mello CV, Clayton DF (1995) Differential induction of the ZENK gene in the avian forebrain and song control circuit after metrazole-induced depolarization. J Neurobiol 26:145-161.

Mello CV, Vicario DS, Clayton DF (1992) Song presentation induces gene expression in the songbird forebrain. Proc Natl Acad Sci USA 89:6818-6822.

Milbrandt J (1987) A nerve growth factor-induced gene encodes a possible transcriptional regulatory factor. Science 238:797-799.

Mishkin M (1978) Memory in monkeys severely impaired by combined but not by separate removal of amygdala and hippocampus. Nature 273:297-298.

Nikolaev E, Kaminska B, Tischmeyer W, Matthies H, Kaczmarek L (1992) Induction of expression of genes encoding transcription factors in the rat brain elicited by behavioral training. Brain Res Bull 28:479-484.

O'Hara BF, Young KA, Watson FL, Heller HC, Kilduff TS (1993) Immediate early gene expression in brain during sleep deprivation: preliminary observations. Sleep 16:1-7.

Pavlides C, Winson J (1989) Influences of hippocampal place cell firing in the awake state on the activity of these cells during subsequent sleep episodes. J Neurosci 9:2907-2918.

Paxinos G (1995) The rat nervous system, Ed 2. San Diego: Academic.

Paxinos G, Watson C (1997) The rat brain in stereotaxic coordinates, Ed 3. San Diego: Academic.

Pearlman C, Becker M (1974) REM sleep deprivation impairs bar-press acquisition in rats. Physiol Behav 13:813-817.

Petersohn D, Schoch S, Brinkmann DR, Thiel G (1995) The human synapsin II gene promoter. Possible role for the transcription factor zif268/egr-1, polyoma enhancer activator 3 , and AP2. J Biol Chem 270:24361-24369.

Poe GR, Nitz DA, McNaughton BL, Barnes CA (2000a) Experiencedependent phase-reversal of hippocampal neuron firing during REM sleep. Brain Res 855:176-180.

Poe GR, Teed RG, Insel N, White R, McNaughton BL, Barnes CA (2000b) Partial hippocampal inactivation: effects on spatial memory performance in aged and young rats. Behav Neurosci 114:940-949.

Pompeiano M, Cirelli C, Tononi G (1992) Effects of sleep deprivation on fos-like immunoreactivity in the rat brain. Arch Ital Biol 130:325-335.

Pompeiano M, Cirelli C, Tononi G (1994) Immediate-early genes in spontaneous wakefulness and sleep: expression of c-fos and NGIF-A mRNA protein. J Sleep Res 3:80-96.

Ribeiro S, Goyal V, Mello CV, Pavlides C (1999) Brain gene expression during REM sleep depends on prior waking experience. Learn Mem 6:500-508.

Rogan MT, Staubli UV, LeDoux JE (1997) Fear conditioning induces associative long-term potentiation in the amygdala. Nature 390:604-607.

Scoville WB, Milner B (1957) Loss of recent memory after bilateral hippocampal lesions. J Neurol Neurosurg Psychiatry 20:11-21.

Shiromani PJ, Kilduff TS, Bloom FE, McCarley RW (1992) Cholinergically induced REM sleep triggers Fos-like immunoreactivity in dorsolateral pontine regions associated with REM sleep. Brain Res 580:351-357.

Siegel JM (2001) The REM sleep-memory consolidation hypothesis. Science 294:1058-1063.

Smith C (1996) Sleep states, memory processes, and synaptic plasticity. Behav Brain Res 78:49-56.

Smith C, Butler S (1982) Paradoxical sleep at selective times following training is necessary for learning. Physiol Behav 29:469-473

Smith C, Kelly G (1988) Paradoxical sleep deprivation applied two days after end of training retards learning. Physiol Behav 43:213-216.

Smith C, Rose GM (1996) Evidence for a paradoxical sleep window for place learning in the Morris water maze. Physiol Behav 59:93-97.

Squire LR (1992) Memory and the hippocampus: a synthesis from findings with rats, monkeys, and humans. Psychol Rev 99:195-231.

Stickgold R, James L, Hobson JA (2000a) Visual discrimination learning requires sleep after training. Nat Neurosci 3:1237-1238.

Stickgold R, Whidbee D, Schirmer B, Patel V, Hobson JA (2000b) Visual discrimination task improvement: a multi-step process occurring during sleep. J Cogn Neurosci 12:246-254.

Stickgold R, Hobson JA, Fosse R, Fosse M (2001) Sleep, learning, and dreams: off-line memory reprocessing. Science 294:1052-1057.

Sukhatme VP, Cao XM, Chang LC, Tsai-Morris CH, Stamenkovich D, Ferreira PC, Cohen DR, Edwards SA, Shows TB, Curran T, Le Beau MM, Adamson ED (1988) A zinc finger-encoding gene coregulated with c-fos during growth and differentiation, and after cellular depolarization. Cell 53:37-43.

Thiel G, Schoch S, Petersohn D (1994) Regulation of synapsin I gene expression by the zinc finger transcription factor zif268/egr-1. J Biol Chem 269:15294-15301. 
Thomson D (1982) Spectrum estimation and harmonic analysis. Proc IEEE 70:1055-1096.

Timo-Iaria C, Negrao N, Schmidek W R, Hoshino K, Lobato de Menezes CE, Leme da Rocha T (1970) Phases and states of sleep in the rat. Physiol Behav 5:1057-1062.

Wallace CS, Withers GS, Weiler IJ, George JM, Clayton DF, Greenough WT (1995) Correspondence between sites of NGFI-A induction and sites of morphological plasticity following exposure to environmental complexity. Brain Res Mol Brain Res 32:211-220.

Williams JA, Comisarow J, Day J, Fibiger HC, Reiner PB (1994) Statedependent release of acetylcholine in rat thalamus measured by in vivo microdialysis. J Neurosci 14:5236-5242.
Winson J (1985) Brain and psyche. New York: Anchor.

Winson J, Dahl D (1986) Long-term potentiation in dentate gyrus: induction by asynchronous volleys in separate afferents. Science 234:985-988.

Wisden W, Errington ML, Williams S, Dunnett SB, Waters C, Hitchcock D, Evan G, Bliss TV, Hunt SP (1990) Differential expression of immediate early genes in the hippocampus and spinal cord. Neuron 4:603-614.

Worley PF, Bhat RV, Baraban JM, Erickson CA, McNaughton BL, Barnes CA (1993) Thresholds for synaptic activation of transcription factors in hippocampus: correlation with long-term enhancement. J Neurosci 13:4776-4786. 